\title{
Topical application of Jaungo in atopic dermatitis patients: study protocol for a randomized, controlled trial
}

\author{
Younghee Yun ${ }^{1}$, Youme Ko ${ }^{2}$, Jin-Hyang Ahn, Bo-Hyoung Jang ${ }^{2}$, Kyuseok Kim³ , Seong-Gyu Ko ${ }^{2}$ and Inhwa Choi ${ }^{*}$
}

\begin{abstract}
Background: Atopic dermatitis (AD) is a common pruritic inflammatory skin disease with increasing prevalence. It can manifest with many different clinical phenotypes; however, in its chronic stage, hyperpigmentation, excoriation, lichenification, and dryness are the main symptoms. Jaungo comprises two herbs, Lithospermi radix and Angelica gigantis radix, and three carrier oils, and is an approved herbal ointment for xerosis cutis in Korea. In past studies, we demonstrated that Jaungo had anti-inflammatory and antiallergic activity in in vitro and in vivo AD models; however, there are few relevant randomized controlled clinical trials on Jaungo in AD.
\end{abstract}

Methods/design: A randomized, double-blind, placebo-controlled, single-center, phase lla clinical trial was designed to investigate the safety, preliminary efficacy, and dose response of Jaungo in AD. The study protocol was approved by the Institutional Review Boards of the Kyung Hee University Korean Medicine Hospital (No. KOMCIRB-160617-HR-027) and the Korea Food and Drug Administration (No. 30907). The study aims to enroll 34 AD patients to be randomly distributed among three parallel groups: treatment 1, treatment 2, and the placebo group. Treatment group 1 applies Jaungo twice a day, while treatment group 2 applies Jaungo and the placebo ointment once a day, separately, and the placebo group applies the placebo ointment twice a day, for a total of 3 weeks each. Participants will be evaluated for eczema before and after the application of the ointments based on several parameters including the Eczema Area and Severity Index, the SCORing of Atopic Dermatitis Index, the Dermatology Life Quality Index, transepidermal water loss, total IgE level, eosinophil count, and IL-17, IL-22, and IFN- levels.

Discussion: The trial is currently ongoing and the enrollment of subjects has been initiated. There is an urgent need to develop a drug for the treatment of dry, hyperpigmented, scaly, and thickened skin in chronic-stage AD. This study will determine the efficacy and safety of Jaungo in AD, providing evidence for specific AD symptoms treated by Jaungo.

Trial registration: Clinical Trials.gov, identifier: NCT02900131. Registered on 2 September 2016. Korea Clinical Research Information Service, identifier: KCT0002060. Registered on 22 July 2016.

Keywords: Dermatitis, Atopic, Jaungo, Randomized controlled trial, Phytotherapy, Administration, Application, Topical

\section{Background}

Atopic dermatitis (AD) is a common pruritic inflammatory skin disease with increasing prevalence in industrialized countries. The prevalence of $\mathrm{AD}$ is $5-20 \%$ worldwide [1]. Altered immunity and reduced barrier function are fundamental to the development of $\mathrm{AD}$ [2], and the heterogeneous pathologic abnormalities observed in this patient

\footnotetext{
* Correspondence: inhwajun@khnmc.or.kr

'Department of Korean Dermatology, Kyung Hee University Hospital at Gangdong, College of Korean Medicine, Kyung Hee University, Seoul, South Korea

Full list of author information is available at the end of the article
}

population may contribute to the different clinical features seen in each patient. In the acute phase, $\mathrm{AD}$ manifests as erythema, microvesiculation, exudation, and crusting; however, in the chronic phase, the main symptoms of $\mathrm{AD}$ include hyperpigmentation, excoriation, and dryness. Additionally, severe itch can induce lichenification in the chronic stage owing to protracted rubbing of the skin [3].

The most effective therapy for $\mathrm{AD}$ includes short-term treatment of flares and a long-term maintenance approach to skin care designed to prevent or minimize flares [4]. Topical anti-inflammatory glucocorticoids (GCs) are 
standard therapy; however, long-term use of GCs carries the risk of side effects leading to the need for development of a therapeutic nonsteroidal topical agent.

Jaungo is an herbal ointment for xerosis cutis, frostbite, miliaria, anal fissures, and rhus dermatitis and approved by the Korea Food and Drug Administration (KFDA). It is composed of two herbs and three carrier oils: Lithospermi radix, Angelica gigantis radix, sesame seed oil, bees wax, and swine oil. Previous studies have demonstrated the anti-inflammatory and antiallergic activity of Jaungo in in vitro and in vivo AD models [5-7]. Additionally, a case series in 1999 reported the effect of Jaungo on AD [8], and two more recent studies reported the effects of Jaungo on both radiation dermatitis in breast cancer patients and cutaneous leishmaniasis $[9,10]$. However, there are few relevant randomized controlled clinical trials on Jaungo for the treatment of AD.

The experimental focus of this trial is to evaluate the safety, preliminary efficacy, and dose response of Jaungo in patients having mild-to-moderate $\mathrm{AD}$ with hyperpigmentation, excoriation, lichenification, and dryness.

\section{Methods}

\section{Study setting and design overview}

This study will be conducted at the Kyung Hee University Korean Medicine Hospital in Seoul, Korea. The study is designed as a randomized, double-blind, placebo-controlled, single-center, phase IIa clinical trial to investigate the safety, preliminary efficacy, and dose response of Jaungo for treatment of AD. The study aims to enroll 34 patients with $\mathrm{AD}$. Participants fulfilling the eligibility criteria will be selected and randomly distributed into three parallel groups: treatment 1 , treatment 2 , and the placebo group. Treatment group 1 applies Jaungo twice a day; treatment group 2 applies Jaungo and placebo ointments once a day, separately; and the placebo group applies placebo ointment twice a day, for a total of 3 weeks each. Participants will be evaluated for AD before and after applying ointment (see Figs. 1 and 2). A completed Standard Protocol Items: Recommendations for Interventional Trials (SPIRIT) Checklist for the trial is available (see Additional file 1).

\section{Eligible criteria}

\section{Inclusion criteria}

Patients will be eligible if they (1) have received a diagnosis of AD according to Hanifin's and Rajka's criteria,

(2) are men or women aged 5 years to below 65 years,

(3) have mild-to-moderate AD symptoms according to the objective SCORing Atopic Dermatitis (SCORAD) Index (score $\leq 40$ ), and (4) have scores of excoriation, lichenification, and dryness in the SCORAD Index of at least 1 each, or a sum of 3 or more.

\section{Exclusion criteria}

The exclusion criteria are as follows: (1) lesions with oozing, (2) oral administration of corticosteroids, immunosuppressants, or antibiotics 4 weeks prior to study entry, (3) administration of topical GCs, immunosuppressants, or antibiotics, or phototherapy, 2 weeks prior to study entry, (4) burn or trauma on the lesions, (5) allergic to Jaungo, or its components, including Lithospermi radix, Angelica gigantis radix, sesame seed oil, bees wax, and swine oil, (6) active skin diseases without AD, (7) renal or liver dysfunction, (8) other uncontrolled chronic diseases, (9) pregnancy or breastfeeding, (10) participation in other clinical trials within 1 month of enrollment, (11) inability to understand the written consent or to engage in this study due to mental impairment or other emotional or mental problems, and (12) judgment by experts that the potential subject's participation is inappropriate.

Exclusion will be primarily based on information provided by the patient. Additionally, patients will undergo blood tests for aspartate aminotransferase (AST), alanine aminotransferase (ALT), blood urea nitrogen (BUN), creatinine $(\mathrm{Cr})$, complete blood cell count/differential count $(\mathrm{CBC} \mathrm{D} / \mathrm{C})$, erythrocyte sedimentation rate (ESR), human chorionic gonadotropin (hCG), and vital signs before the trial to ensure that the patients do not suffer from the listed diseases. A patient with liver dysfunction is defined as having ALT and AST values 2.5 times the upper limit of normal values (over $125 \mathrm{mg} / \mathrm{dL}$ for men and over $87.5 \mathrm{mg} / \mathrm{dL}$ for women). A patient with renal dysfunction was defined as having a serum $\mathrm{Cr}$ value over $2.0 \mathrm{mg} / \mathrm{dL}$.

\section{Intervention overview}

Participants will apply Jaungo or placebo ointment for 3 weeks twice a day. The defined daily doses of Jaungo should be determined by the KFDA guidelines for specifications and analytical procedures of drug products; however, no pilot study has been performed. Therefore, we are utilizing the previously approved defined daily doses of Jaungo for treatment of xerosis cutis. Of note, Jaungo is given at a dose twice the strength of topical GCs; however, its antiinflammatory activity is much less than that of topical GCs.

The fingertip unit (FTU) measurement will be utilized for participants to determine the required amount of the Jaungo to be used (Table 1). One FTU, approximately $0.5 \mathrm{~g}$, is equal to the amount of ointment that is scooped out from the ointment pot with an adult fingertip (Fig. 3). The treatment area for the application of 1 FTU is equal to an area of the skin the size of a flat adult hand with fingers together. Participants will receive instructions for the specific amount of ointment to apply depending on their $\mathrm{AD}$ lesions.

\section{Experimental medicine (Jaungo)}

Jaungo ointment (Hanpoong Pharm and Foods, Co., Ltd., Wanju, Korea) was produced according to Korea 


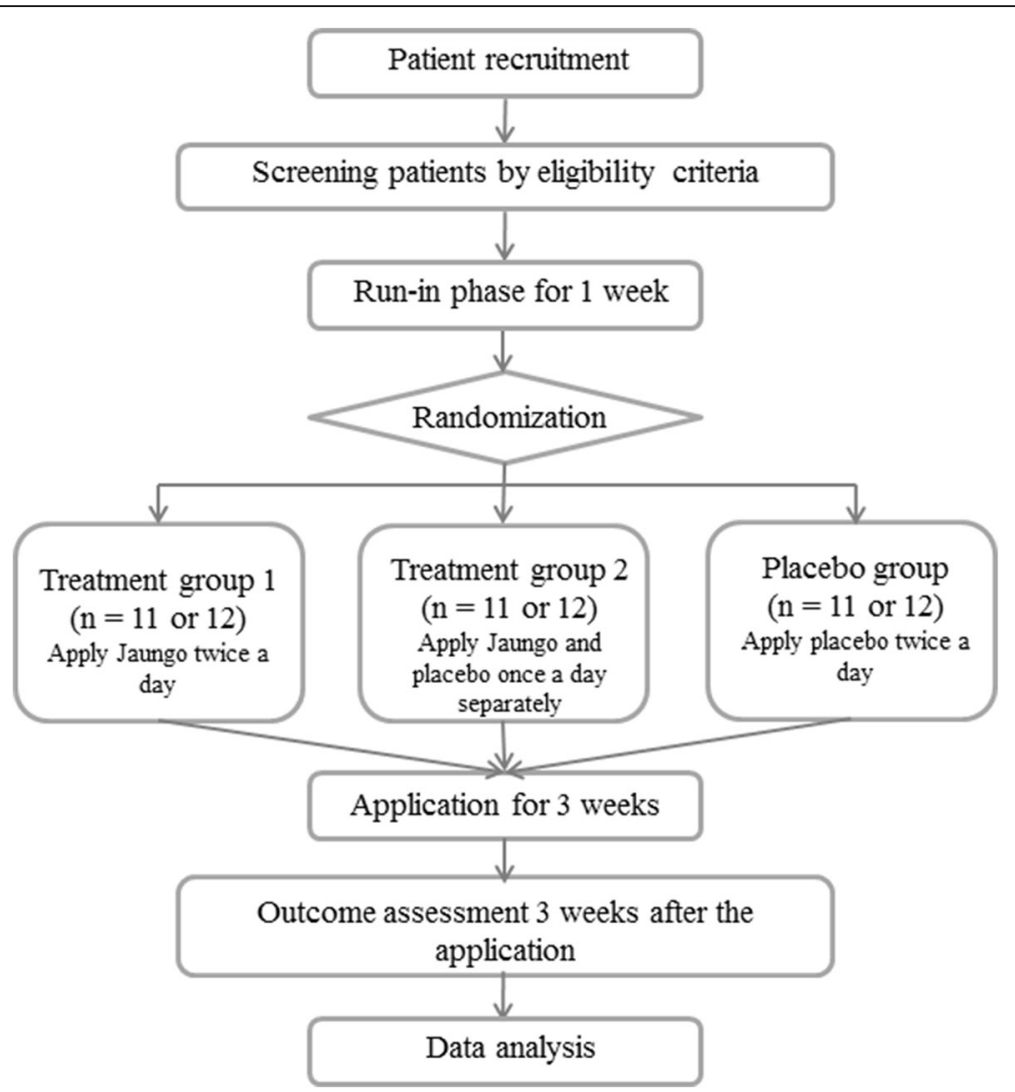

Fig. 1 Study flow chart

\begin{tabular}{|c|c|c|c|c|}
\hline & \multicolumn{4}{|c|}{ STUDY PERIOD } \\
\hline & Enrolment & Allocation & Intervention & Close-out \\
\hline & Visit1 & Visit2 & & Visit3 \\
\hline TIMEPOINT ${ }^{* *}$ & $-7 \sim-2$ day & 0 & 21 days & 21 day \\
\hline \multicolumn{5}{|l|}{ ENROLMENT: } \\
\hline \multirow{2}{*}{$\begin{array}{l}\text { Eligibility screen } \\
\text { Informed consent }\end{array}$} & $x$ & & & \\
\hline & $\mathrm{x}$ & & & \\
\hline \multirow[t]{2}{*}{ Enrollment } & & $x$ & & \\
\hline & & $x$ & & \\
\hline \multicolumn{5}{|l|}{ INTERVENTIONS: } \\
\hline \multicolumn{5}{|l|}{ Treatment Group 1} \\
\hline \multicolumn{5}{|l|}{ Treatment Group 2} \\
\hline \multicolumn{5}{|l|}{ Placebo Group } \\
\hline \multicolumn{5}{|l|}{ ASSESSMENTS: } \\
\hline $\begin{array}{r}\text { Efficacy } \\
\text { outcomes }^{1}\end{array}$ & & $x$ & & $x$ \\
\hline Safety outcomes ${ }^{2}$ & $x$ & $x$ & & $\mathrm{x}$ \\
\hline
\end{tabular}

Fig. 2 The schedule of enrollment, interventions, and assessments 
Table 1 Fingertip unit measurement (FTU) required to cover a specific skin area

\begin{tabular}{llll}
\hline Area of skin to be treated & Palm-sized & FTUs each dose (adults) & FTUs each dose (child) \\
\hline A hand and fingers (front and back) & About 2 palms & 2 FTUs (1 g) & 1 FTUs (0.5 g) \\
Front of chest and abdomen & About 14 palms & 14 FTUs (7 g) & 7 FTUs (3.5 g) \\
Back and buttocks & About 14 palms & 14 FTUs (7 g) & 10 FTUs (5 g) \\
Face and neck & About 5 palms & 5 FTUs (2.5 g) & 4 FTUs (2 g) \\
An entire arm and hand & About 8 palms & 8 FTUs (4 g) & 5 FTUs (2.5 g) \\
An entire leg and foot & About 16 palms & 16 FTUs (8 g) & 9 FTUs (4.5 g) \\
\hline
\end{tabular}

Good Manufacturing Practice standards. Jaungo was approved for the treatment of xerosis cutis, frostbite, miliaria, anal fissure, and rhus dermatitis on 11 September 2014 , by the KFDA. It is licensed to be applied to skin lesions one or more times per day.

Jaungo is composed of two herbs and three carrier oils: $72.7 \mathrm{mg}$ of Lithospermi radix, $60.6 \mathrm{mg}$ of Angelica gigantis radix, $60.1 \mathrm{mg}$ of sesame seed oil, $242.4 \mathrm{mg}$ of bees wax, and $18.2 \mathrm{mg}$ of swine oil based on a total $1 \mathrm{~g}$ of ointment. It is a red-colored ointment with an herbal fragrance. The characteristic red color is due to the Lithospermi radix, and the distinctive aroma is due to the flavor components of bees wax and Angelica gigantis radix.

\section{Placebo medicine}

Hanpoong Pharm and Foods Co., Ltd., developed a homogenous ointment that is red in color with an herbal fragrance made with $698.1 \mathrm{mg}$ sesame seed oil, $279.2 \mathrm{mg}$ bees wax, $21 \mathrm{mg}$ swine oil, $1.2 \mathrm{mg}$ red iron oxide, and $0.5 \mathrm{mg}$ ginseng-flavored extract based on a total $1 \mathrm{~g}$ of ointment. Substances, content, and preparative methods of the placebo ointment were approved by KFDA. The placebo ointment is very similar to the experimental medicine Jaungo in color, form, weight, and odor.

\section{Intervention discontinuation}

Participants may discontinue the trial in the following cases: violation of the inclusion/exclusion criteria, violation of combinational medications, the occurrence of serious adverse events, request by participant due to side

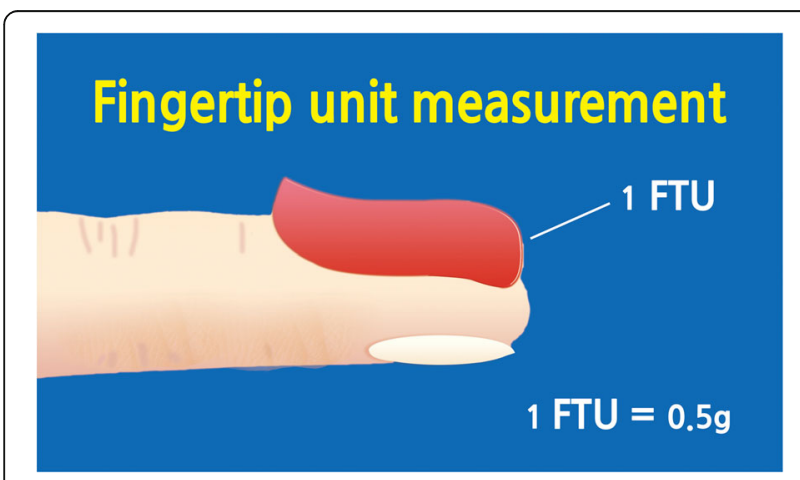

Fig. 3 Fingertip unit measurement effects, determination by the investigator due to side effects, systematic disease discovered during the trial, request of participant or their guardian to discontinue, the need for surgery or hospitalization due to accidents or diseases, pregnancy, worsening of atopic dermatitis, continuation judged inappropriate by the investigator.

\section{Early closure}

If participant $\mathrm{AD}$ symptoms disappear, the investigator may terminate the case early after discussion with the sponsors and the statistician.

\section{Adherence assessment}

Adherence will be assessed by a container count at visit 3. Participants will bring in experimental medications at visit 3, more than $80 \%$ use of experimental medication will be defined as "good adherence," and less than $80 \%$ use will be defined as "poor adherence." Because the amount of ointment applied by each participant will be different according to the extent of the involved lesion, adherence will be calculated according to the dose of ointment distributed for each participant.

\section{Combination medications}

Different medications for $\mathrm{AD}$, such as orally administered antihistamines, GCs, and immunosuppressants, will not be allowed throughout the study period; however, after discussion with the investigators, patients can self-administer antihistamines orally for controlling pruritus. Patients will also be allowed to use emollients, lotions, and ointments that do not contain GCs during the study. Additional herbal prescriptions, acupuncture treatments, or therapeutic interventions by other clinicians will not be allowed during the study period.

These restrictions are based on the information provided by the patient, and researchers will have to evaluate whether the information provided will affect AD. Drugs taken by each participant will be recorded at every visit, and participants will be asked to notify us of any changes in their medication/supplement regimen. If the participant received medications that may affect $A D$, they will be excluded from the study. 


\section{Outcomes}

\section{Primary outcome measurement}

The primary outcome will be measured by changes in the Eczema Area and Severity Index (EASI) before and after 3 weeks' application of experimental medications. Key signs of eczema, including erythema (E), infiltration/population (I), excoriation (Ex), and lichenification (L), will be assessed on a numeric scale of 0 to 3 . Then, the percentage of area involved for each of the four body regions will be assigned a proportional score from 0 to 6 : $0=$ no eruption; $1=<10 \% ; 2=<10-29 \% ; 3=<30-49 \% ; 4=<50-$ $69 \%$; $5=<70-89 \%$; and $6=>90-100 \%$. Finally, an established multiplier based on the age of the patient will be used. Please refer to the following formula for patients over 8 years of age. The multiplier for patients under 8 years of age is indicated in parentheses and in italics:

Upper Limbs $[(\mathrm{E}+\mathrm{I}+\mathrm{Ex}+\mathrm{L}) \times$ Area $\times 0.2(0.2)]+$ Lower Limbs $[(\mathrm{E}+\mathrm{I}+\mathrm{Ex}+\mathrm{L}) \times$ Area $\times 0.4(0.3)]+$ Trunk Limbs $[(\mathrm{E}+\mathrm{I}+\mathrm{Ex}+\mathrm{L}) \times$ Area $\times 0.3(0.3)]+$ Head/Neck $[(\mathrm{E}+\mathrm{I}+$ $\mathrm{Ex}+\mathrm{L}) \times$ Area $\times 0.1(0.2)][11]$.

\section{Secondary outcome measurements}

Secondary outcome measurements will include SCORAD score [12], transepidermal water loss (TEWL) using TM 300 [13], the Dermatology Life Quality Index (DLQI) [14], total IgE, eosinophil count, and interleukin (IL)-17, IL-22, and interferon (IFN) $-\gamma$ [15]. These parameters will be measured before and after 3 weeks' application of experimental medications. The consumption of orally administered antihistamines and usage of emollients, lotions, and ointments that do not contain GCs during the study will be checked.

\section{Safety outcome measurements}

Prior to randomization and immediately after treatment completion, we will perform the following tests on all participants: CBC D/C, AST/ALT, BUN/Cr, ESR, and vital signs. These tests will serve to exclude participants who have serious illnesses or abnormal liver, kidney, or other organ function. Additionally, subjective dermal tolerability assessment and the Draize score will be assessed after completion of the study [16].

Listed adverse events verified by the KFDA for Jaungo include allergic skin reactions including rash and pruritus. Investigators will assess the incidence and intensity of adverse events. In addition, a causality evaluation of adverse drug reactions will be discussed and documented in Case Report Forms.

\section{Sample size}

This study aims to investigate the efficacy, safety, and dose response for the approved drug, Jaungo, with a new indication. Jaungo is already approved for use in xerosis cutis, frostbite, miliaria, anal fissures, and rhus dermatitis. There are no previous clinical trials of the use of Jaungo in AD. Following the study by Julious [17], we considered the practical feasibility, the possible expectation of the mean and standard deviation, regulations of the KFDA, and risks which fail to detect the difference among the study groups to determine sample size. From this, we decided that 12 patients per group were needed.

\section{Recruitment}

Participants will be recruited by bulletin board advertisements and the online homepages of Kyung Hee University Korean Medicine Hospital, Kyung Hee University Hospital at Gangdong, and Kyung Hee University in Seoul, Korea. Respondents will be contacted by clinical trial coordinators to determine their eligibility via a telephone prescreening. If an applicant meets the study criteria, they will be invited to the clinical research center to be examined for eligibility.

\section{Randomization and allocation}

Before assigning randomization, all participants will be informed that they will be distributed into one of three groups. Thirty-four participants will be randomized into three groups in a 1:1:1 ratio, using block randomization with a block size of 3 or 6 , where 11-12 participants are planned for each group. Randomization distribution will occur on the second visit to the clinical research center. Participants will be assigned random numbers via a web-based random number generator system developed by an independent expert on web programming using Django Web Framework (https://www.djangoproject.com). The clinical pharmacy will be supplied with the experimental and placebo ointments in identical containers labeled with random numbers, which correspond to the participant's assigned number, and "for morning use" or "for evening use." The participant's randomly assigned number will dictate which ointments the clinical pharmacist will supply to them.

\section{Blinding}

To ensure blinding, a matched placebo ointment identical in color, texture, weight, and odor will be utilized and stored in a container identical to that of Jaungo. The Jaungo and placebo ointments will be labeled with a random number and "for morning use" or "for evening use" in an identical form. Treatment group 1 will receive two containers of Jaungo, one labeled with "for morning use" and the other "for evening use," treatment group 2 will receive one container of Jaungo and one container of placebo ointment where one of the containers is labeled with "for morning use" and the other "for evening use." The placebo group will receive two containers of placebo ointment, one labeled with "for morning use" and the other "for evening use." The clinical pharmacist will 
supply Jaungo, the placebo ointment, or both to the patient depending on the patient's assigned number. If the ointments are lost or destroyed, the clinical pharmacist will supply the patient with extra ointment labeled in an identical manner to maintain blinding.

In this trial, treatment distribution will remain unknown to the participants, investigators, outcomes assessors, statistician, and other staff until the end of the study. The randomization list and blinding codes will be kept strictly confidential. Access to the randomization list will be limited to the Clinical Research Organization (CRO) staff and the list will only be opened as a standard operating procedure. In the event that the randomization list has to be opened immediately, owing to an urgent situation pertaining to serious adverse events, the primary investigator must report the incident to the CRO. Participants will remain blinded to their respective treatment group until the final visit of the last randomized participant. The success of blinding will be assessed at each participant's final visit. Researchers who are blinded to the distribution data will perform the outcome assessment. The blinding procedure will also be verified by the authorized CRO.

\section{Data collection, management, quality control and monitoring}

To maintain the quality of this trial, monitoring and data management including data collection, validation, and completion will be conducted by the Institute of Safety and Effectiveness Evaluation for Korean Medicine (ISEE), a CRO located in Seoul, Korea. To ensure that outcome assessments are of a high standard in accordance with the trial protocol, the investigator and the assistants will attend a 6-h training workshop prior to the initiation of the trial. The investigator and the assistants will also be provided with a written protocol and standard operating procedure documents. All the data will be checked regularly by clinical trial coordinators from ISEE.

\section{Statistical analysis}

The primary hypothesis is that topical application of Jaungo is more effective than the placebo ointment for treating AD. Baseline data collected on each participant at randomization in the trial will be used to describe the population of patients, assess comparability of treatment groups, achieve balanced randomization, adjust for possible confounding prognostic factors, and undertake subgroup analyses. The baseline characteristics of the three groups, including sex, age, duration of $\mathrm{AD}$, total $\mathrm{IgE}$ level, and SCORAD score will be compared. Analysis of variance (ANOVA) will be used to investigate differences in continuously scaled variables, and chi-squared tests will be used to identify significant variations in proportions across treatment groups.
We will also compare the efficacy of Jaungo and placebo ointments based on the change in primary outcome, or EASI score, from day 0 to the end of the study period ( 3 weeks). Mean differences in the EASI score from baseline value to the end of treatment will be compared using ANOVA among the three groups. A paired Student's $t$ test will be used to compare the mean change within a group. Secondary outcomes (SCORAD score, TEWL, DLQI, total IgE, eosinophil count, IL-17, IL-22, and IFN- $\gamma$ ) will also be utilized to compare the efficacy of Jaungo and placebo ointments in the same manner as the primary outcome/EASI score. To evaluate safety, the Draize score and adverse events will be presented in a descriptive manner.

Analyses will be performed for two populations: (1) an intention-to-treat population consisting of all randomized participants who have at least one measurable outcome to report following treatment (missing data are replaced with the last observation values) and (2) a per-protocol population including only participants without major protocol deviations. All data will be descriptively analyzed. All main analyses will be based on the intention-to-treat population and conducted using the last observation carried forward imputation method. Statistical analyses will be conducted in a blind manner by an independent statistician and performed using the SPSS 21 (IBM Inc., Armonk, NY, USA), where the level of significance is established at $\alpha=0.05$.

\section{Discussion}

$\mathrm{AD}$ is an eczematous disorder that manifests with erythema, microvesiculation, exudation and crusting in its acute phase, and dry, red, scaly skin in its chronic phase [3]. Treatment guidelines for AD focus on the acute stage of the disease which includes emollients and topical GCs. Topical GCs are the mainstay treatment for AD flare-ups, and in many cases AD can be controlled with topical corticosteroids or topical calcineurin inhibitors; however, some chronic AD patients require other treatment options due to GC side effects.

Jaungo is an approved herbal ointment for xerosis cutis, frostbite, miliaria, anal fissures, and rhus dermatitis in Korea. Topical application of Jaungo was found to have anti-inflammatory and antiallergic activity in our previous in-vivo and in-vitro studies [5-7]. Based on these findings, we designed a phase IIa clinical study to determine the clinical efficacy of Jaungo and its possible treatment mechanism in chronic-stage AD. This preliminary and exploratory study is critical for future confirmatory clinical trials, as no information exists for Jaungo's application dose, frequency, and length of time that it should be administered in AD.

One of the important issues that we had to consider in this study was the blindness between the experimental drug, Jaungo, and the placebo ointment. Jaungo has a 
unique color and aroma. The characteristic red color is due to the Lithospermi radix, and its distinctive aroma is due to the flavor components of bees wax and Angelica gigantis radix. Red iron oxide and ginseng-flavored extract were used to manufacture the placebo ointment. Substances, composition, and manufacturing methods of the placebo ointment were approved by the KFDA. Additionally, to conceal the distribution data from researchers and participants, a web-based random number generator system was developed by an independent expert on web programming using Django Web Framework.

Limitations of this study are that it involves only 34 participants in a single center. This could potentially influence the generalizability of the study results. However, this study was designed to find the most responsive AD symptoms, to establish the proper application dose and frequency of Jaungo, and to identify the possible mechanism of action utilized by Jaungo for further study. In conclusion, this exploratory study will contribute to establishing Jaungo's efficacy, safety, and dose response in AD.

\section{Trial status}

The trial is currently ongoing. Enrollment of subjects has been initiated.

\section{Additional file}

Additional file 1: SPIRIT Checklist. (DOC $130 \mathrm{~kb}$ )

\begin{abstract}
Abbreviations
AD: Atopic dermatitis; ALT: Alanine aminotransferase; ANOVA: Analysis of variance; AST: Aspartate aminotransferase; BUN: Blood urea nitrogen; CBC D/ C: Complete blood cell count differential count; Cr: Creatinine; CRO: Clinical Research Organization; DLQI: Dermatology Life Quality Index; EASI: Eczema Area and Severity Index; ESR: Erythrocyte sedimentation rate; FTU: Fingertip unit; hCG: Human chorionic gonadotropin; ISEE: Institute of Safety and Efficacy Evaluation; KFDA: Korea Food and Drug Administration; SCORAD: SCORing Atopic Dermatitis; TEWL: Transepidermal water loss
\end{abstract}

\section{Acknowledgements}

Not applicable.

\section{Funding}

This study is supported by the Traditional Korean Medicine R\&D program funded by the Ministry of Health and Welfare through the Korea Health Industry Development Institute (H12C1889 and HI13C0530). The funder had no further role in the study design, data collection, analysis and interpretation of data, writing of the report, or the decision to submit the paper for publication.

\section{Availability of data and materials}

The datasets generated and/or analyzed during this study will be available upon reasonable request made to the corresponding author.

\section{Authors' contributions}

YY, YK, and JA made substantial contributions to the conception and design of this study as well as to the drafting of the manuscript. BJ and KK are biostatisticians and they designed and supervised the data management, statistical analysis, and randomization and blinding methodology. SK and IC are the general supervisors for this research and participated in both the study design and critical revision of the manuscript. All authors read and approved the final manuscript.

\section{Authors' information}

Not applicable.

\section{Competing interests}

The authors declare that they have no competing interests.

\section{Consent for publication}

Not applicable.

\section{Ethics approval and consent to participate}

This study protocol version 1.1 was approved by the Institutional Review Board (IRB) of the Kyung Hee University Korean Medicine Hospital (No. KOMCIRB-160617-HR-027) and the KFDA (No. 30907). Voluntary informed consent will be obtained from parents or legal guardians of minor patients, and from adult patients. This study will be conducted in accordance with the relevant laws and regulations of the KFDA.

\section{Publisher's Note}

Springer Nature remains neutral with regard to jurisdictional claims in published maps and institutional affiliations.

\section{Author details}

${ }^{1}$ Department of Korean Dermatology, Kyung Hee University Hospital at Gangdong, College of Korean Medicine, Kyung Hee University, Seoul, South Korea. ${ }^{2}$ Department of Preventive Medicine, Graduate School, Kyung Hee University, Seoul, Republic of Korea. ${ }^{3}$ Department of Ophthalmology, Otolaryngology and Dermatology of Korean Medicine, College of Korean Medicine, Kyung Hee University, Seoul, South Korea.

Received: 12 October 2016 Accepted: 25 March 2017

Published online: 12 April 2017

\section{References}

1. Williams H, Robertson C, Stewart A, Ait-Khaled N, Anabwani G, Anderson R, Asher I, Beasley R, Bjorksten B, Burr M, et al. Worldwide variations in the prevalence of symptoms of atopic eczema in the International Study of Asthma and Allergies in Childhood. J Allergy Clin Immunol. 1999;103(1 Pt 1):125-38.

2. Terui T. Analysis of the mechanism for the development of allergic skin inflammation and the application for its treatment: overview of the pathophysiology of atopic dermatitis. J Pharmacol Sci. 2009;110(3):232-6.

3. Pugliarello S, Cozzi A, Gisondi P, Girolomoni G. Phenotypes of atopic dermatitis. J Dtsch Dermatol Ges. 2011;9(1):12-20.

4. Walling HW, Swick BL. Update on the management of chronic eczema: new approaches and emerging treatment options. Clin Cosmet Investig Dermatol. 2010;3:99-117.

5. Kim IS, Kim DH, Yun CY, Lee JS. A (S)-(+)-decursin derivative, (S)-(+)-3-(3,4dihydroxy-phenyl)-acrylic acid 2,2-dimethyl-8-oxo-3,4-dihydro-2H,8Hpyrano[3,2-g]-chromen-3-yl-ester, attenuates the development of atopic dermatitis-like lesions in NC/Nga mice. Mol Biol Rep. 2013;40(3):2541-8.

6. Son SH, Park KK, Park SK, Kim YC, Kim YS, Lee SK, Chung WY. Decursin and decursinol from Angelica gigas inhibit the lung metastasis of murine colon carcinoma. Phytother Res. 2011;25(7):959-64.

7. Li L, Li W, Jung SW, Lee YW, Kim YH. Protective effects of decursin and decursinol angelate against amyloid beta-protein-induced oxidative stress in the PC12 cell line: the role of Nrf2 and antioxidant enzymes. Biosci Biotechnol Biochem. 2011;75(3):434-42.

8. Higaki S, Kitagawa T, Morohashi M, Yamagishi T. Efficacy of Shiunko for the treatment of atopic dermatitis. J Int Med Res. 1999:27(3):143-7.

9. Kong M, Hwang DS, Lee JY, Yoon SW. The efficacy and safety of Jaungo, a traditional medicinal ointment, in preventing radiation dermatitis in patients with breast cancer: a prospective, single-blinded, randomized pilot study. Evid Based Complement Alternat Med. 2016;2016:9481413.

10. Na-Bangchang K, Ahmed O, Hussein J, Hirayama K, Kongjam P, Aseffa A, Karbwang J. Exploratory, phase II controlled trial of Shiunko ointment local application twice a day for 4 weeks in Ethiopian patients with localized cutaneous leishmaniasis. Evid Based Complement Alternat Med. 2016;2016:5984709.

11. Hanifin JM, Thurston M, Omoto M, Cherill R, Tofte SJ, Graeber M. The Eczema Area and Severity Index (EASI): assessment of reliability in atopic dermatitis. EASI Evaluator Group. Exp Dermatol. 2001;10(1):11-8. 
12. Stalder JF, Taïeb A, Atherton DJ, Bieber P, Bonifazi E, Broberg A, et al. Severity scoring of atopic dermatitis: The SCORAD index: Consensus report of the european task force on atopic dermatitis. Dermatology. 1993;186(1): 23-31. Available from: doi10.1159/000247298.

13. Miteva M, Richter S, Elsner P, Fluhr JW. Approaches for optimizing the calibration standard of Tewameter TM 300. Exp Dermatol. 2006;15(11):904-12.

14. Finlay AY, Khan GK. Dermatology Life Quality Index (DLQI) — a simple practical measure for routine clinical use. Clin Exp Dermatol. 1994;19(3):210-6.

15. Mansouri Y, Guttman-Yassky E. Immune pathways in atopic dermatitis, and definition of biomarkers through broad and targeted therapeutics. J Clin Med. 2015:4(5):858-73.

16. Draize JHWG, Calvery HO. Methods for the study of irritation and toxicity of substances applied topically to the skin and mucous membranes. J Pharmacol Exp Ther. 1944;82:377-90.

17. Julious SA. Sample size of 12 per group rule of thumb for a pilot study. Pharm Stat. 2005;4(4):287-91.

\section{Submit your next manuscript to BioMed Central} and we will help you at every step:

- We accept pre-submission inquiries

- Our selector tool helps you to find the most relevant journal

- We provide round the clock customer support

- Convenient online submission

- Thorough peer review

- Inclusion in PubMed and all major indexing services

- Maximum visibility for your research

Submit your manuscript at www.biomedcentral.com/submit 